\title{
Changes in frequency of spontaneous oscillations in procerebrum correlate to behavioural choice in terrestrial snails
}

\author{
Elena Samarova and Pavel Balaban* \\ Institute of Higher Nervous Activity and Neurophysiology, Russian Academy of Science, Moscow, Russia
}

Edited by:

Yosef Yarom, Hebrew University, Israel

\section{Reviewed by:}

Avraham Susswein, Bar-llan University, Israel

Alan Gelperin, Monell Chemical

Senses Center, USA

\section{${ }^{*}$ Correspondence:}

Pavel Balaban, Institute of Higher Nervous Activity and Neurophysiology, Butlerova 5a, Moscow 117485, Russia. e-mail:balaban@ihna.msk.ru
The aim of our study was to understand functional significance of spontaneous oscillations of local field potential in the olfactory brain lobe of terrestrial snail, the procerebrum (PC). We compared changes in frequency of oscillations in semi-intact preparations from snails trained to percept the same conditioned odor as positive (associated with food reinforcement) or negative (associated with noxious reinforcement). In vivo recordings in freely behaving naïve snails showed a significant decrease of spontaneous PC oscillations frequency during a stage of tentacle withdrawal to odor presentation. In in vitro preparations from naïve snails, a similar decrease in frequency of the PC oscillations to odor presentation was observed. Changes in frequency of the oscillations to cineole presentations in the "aversive" group of snails (demonstrating withdrawal) were much more pronounced than in naïve snails. No significant difference in responses to $5 \%$ and $20 \%$ cineole was noted. Changes in the spontaneous oscillations frequency in the snails trained to respond with positive reaction (approach) to cineole depended on the concentration of the applied odor, and these responses were qualitatively similar to responses of other groups during the first $10 \mathrm{~s}$ of responses to odor, but significantly different (increase in PC oscillations frequency) from the responses of the aversively trained and naïve snails in the interval 11-30 s, which corresponds to the end of the tentacle withdrawal and timing of decision making (approach or escape) in the free behaving snails. Obtained results suggest that frequency of the PC lobe spontaneous oscillations correlate to the choice of behavior in snails: withdrawal (decrease in frequency) or approach (increase in frequency) to the source of odor.

Keywords: invertebrates, odor perception, decision making, neurons

\section{INTRODUCTION}

Olfaction in terrestrial gastropod mollusks (snails and slugs) is an important sensory modality for locating and differentiating odors during feeding and orientation. The olfactory sensory epithelium pads in terrestrial snails and slugs are situated on the tentacle tips (Chase and Croll, 1981; Chase, 1985), and are directly exposed to air currents. Each peripheral olfactory organ consists of an olfactory ganglion connected, on the one hand, to the sensory pad, and, on the other hand, to the central ganglia complex. The olfactory ganglia contain about 20 glomeruli (Chase and Tolloczko, 1986), and represent a second stage of olfactory information processing. Axons of the primary sensory cells are ending either in the digitary extensions or in glomeruli of the tentacle ganglion. A small part of the sensory cells population sends their axons directly to the cerebral ganglion (Chase and Tolloczko, 1986). Thus, there are three parallel pathways from the sensory pad primary neurons used in olfactory processing: to glomeruli, to interneurons of a tentacular ganglion, and direct projections to the cerebral ganglia (Chase and Tolloczko, 1993; Ierusalimsky and Balaban, 2007). The PC lobe is a division of the cerebral ganglion unique to terrestrial slugs and snails that is specialized for the processing (third stage) of olfactory information (Ratte and Chase, 1997). The PC lobe in terrestrial snails and slugs spontaneously produces a periodic slow oscillations of local field potential (Gelperin and Tank, 1990; Chase and Tolloczko, 1993; Kleinfeld et al., 1994; Nikitin and Balaban, 1999,
2000). Oscillatory neuronal activity is common in the olfactory system of both vertebrates (Gray and Skinner, 1988; Laurent and Davidowitz, 1994; Dorries and Kauer, 2000; Lam et al., 2000) and invertebrates (Mellon and Wheller, 1999; Wehr and Laurent, 1999; Nikitin and Balaban, 2005). The role of these oscillations in mechanisms of odor perception and discrimination, however, is yet unclear.

Increasing evidence indicates that the PC lobe is involved in odor learning in Limax and Helix (Nikitin and Balaban, 2000; Watanabe et al., 2008). Bilateral ablation of the PC lobe, either before or after conditioning, resulted in impairment of memory (Kasai et al., 2006). However, ablation of the PC lobe did not affect detection of odors, because the ablated slugs showed normal avoidance to the innately aversive odor of garlic.

It was described in mammals that each receptor cell expresses only one of receptor genes. Moreover, axons of these cells are going to one type of the glomeruli (Buck and Axel, 1991; Mombaerts et al., 1996). However, the next step of odor recognition in the central nervous system (CNS), its categorization as positive or negative for the animal, choice of behaviour (approach or withdrawal) has not been well described yet.

It is well known that molluscs can learn a variety of new odors (Balaban, 2002). Pairing the electric shock with food results in strong aversion to the taste and smell of the conditioned food in the snail Helix (Balaban, 1993). Likewise, the slug Limax tends to 
avoid an area with a strong concentration of the odor that previously had been paired with noxious quinidine sulfate (Sahley et al., 1981). Evidently, behavioural response to odors should in some way be correlated with the procerebral activity involved in olfactory information processing. These include oscillatory dynamics modified by behaviourally relevant odors (Gervais et al., 1996; Kimura et al., 1998; Inoue et al., 2006).

In the present report we describe changes in frequency of the PC lobe spontaneous oscillations in semi-intact preparations from snails trained to percept the same conditioned odor as positive (associated with food reinforcement) or negative (associated with noxious reinforcement). Comparison with changes in frequency of the PC lobe oscillations in free behaving naïve snails is made.

\section{MATERIAL AND METHODS EXPERIMENTAL SUBJECTS}

The experiments were performed both in vivo in adult snails (Helix lucorum L.) and in vitro using semi-intact preparations with intact olfactory nerves and sensory pads on the tip of the tentacles (Figure 1C). The snails were kept in an active state, maintained in a

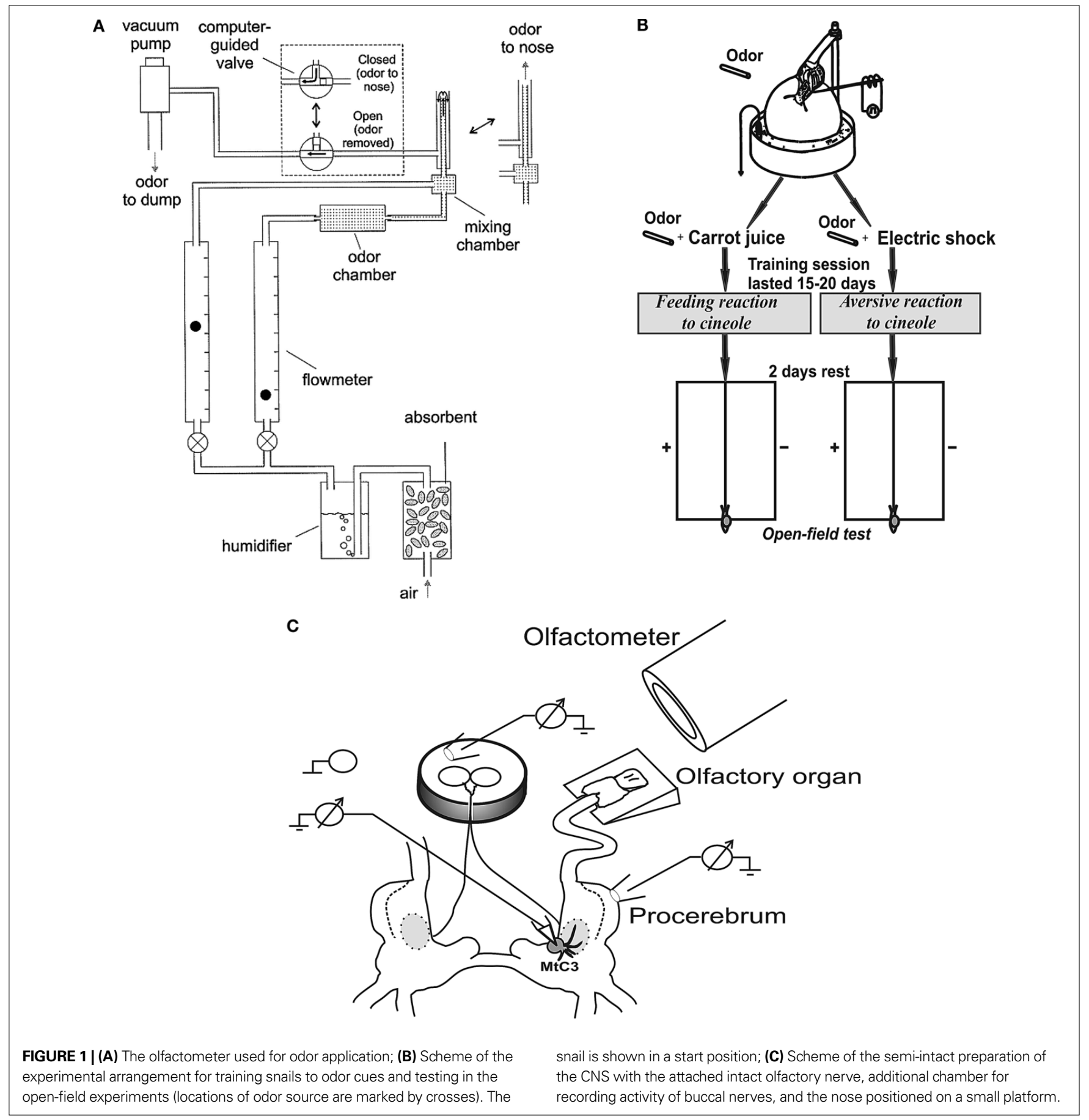


humid environment and were fed cabbage. All efforts were made to minimize pain and quantity of animals. The Ethics Commission of Institute of Higher Nervous Activity, Russian Academy of Sciences, approved the experimental protocol.

\section{ELECTROPHYSIOLOGICAL RECORDING FROM THE PROCEREBRUM AND CNS NEURONS IN VITRO \\ Preparation}

The experiments were performed on semi-intact (nose-brain) preparations of the snail Helix lucorum L. with intact olfactory nerve and sensory epithelium (Figure 1C).

Before dissection, the animals were anaesthetized by injection of an isotonic solution of $\mathrm{MgCl}_{2}$. All efforts were made to minimize pain, the protocol of preparation was approved by Ethics Commission of Institute of Higher Nervous Activity. The composition of the Helix saline used in these experiments was as follows: $100(\mathrm{~mm}) \mathrm{Na}^{+}, 4.2(\mathrm{~mm}) \mathrm{K}^{+}, 7(\mathrm{~mm}) \mathrm{Ca}^{2+}, 4.6(\mathrm{~mm}) \mathrm{Mg}^{2+}$, and $127.4(\mathrm{~mm}) \mathrm{Cl}^{-}$. The cerebral ganglia and $\mathrm{PC}$ were partially de-sheathed, using fine forceps. To remove the thick connective tissue from the PC and to make neurons accessible for microelectrode recording, the sheath was treated by protease (type XIV, Sigma) for $7 \mathrm{~min}$. Then the preparation was washed with large amounts of saline for 20-30 min to remove all the protease from the experimental bath.

The tentacle was pinned by the retractor muscle to the silgard plate higher then the saline level (Figure 1C; Egan and Gelperin, 1981). Sensory epithelium was covered with the tentacle skin flap that was removed at the last moment before the start of experiment.

\section{EXTRACELLULAR RECORDING FROM THE PROCEREBRUM}

Simultaneous extracellular recordings from the PC and cerebrobuccal connective were made as well as intracellular recording of neuronal activity from the neurons from different ganglia of the snail's CNS.

Electrophysiological recordings of the PC activity were performed using suction glass electrodes (inner diameter $\sim 50 \mu \mathrm{m}$ ) filled with Helix saline. The recorded potentials were fed to a microelectrode amplifier set at a bandpass of 0-50 Hz (DL302P, NB-Lab, Russia).

Extracellular recordings of the activity of the cerebro-buccal connective were made using additional small chamber $(6 \mathrm{~mm}$ in diameter) inside of a bigger chamber that contained the cerebral ganglia and the remaining parts of the snail's CNS. Buccal ganglia were placed in a small chamber, the nerves passing through a slit filled with vaseline (Figure 1C). The saline solution in the small bath was not connected with the saline in the big one, while the recording and reference electrodes were placed on the opposite sides of the vaseline wall.

\section{INTRACELLULAR RECORDING FROM THE CNS NEURONS}

We recorded intracellularly activity of the metacerebral neuron 3 (MtC3) (Figure 1C), metacerebral neuron 1 ( MtC1), pedal ventral $5(\mathrm{Pd} 5 \mathrm{v})$, pleural 1 ( $\mathrm{Pl} 1$, in some experiments) using sharp glass microelectrodes filled with $1.5 \mathrm{M} \mathrm{KCl}$ and $1 \mathrm{M} \mathrm{KCH}_{2} \mathrm{COOH}$ (tip resistance 10-50 M $\Omega$ ). The recorded potentials were fed to a microelectrode amplifier set at a bandpass of $0-2 \mathrm{kHz}$. The amplifier output was digitized.

\section{ODOR APPLICATION}

Our previous experiments have demonstrated the snail behavioural responses to a number of volatile monomolecular chemicals, such as cineole, isoamyl acetate (IAA) and benzaldehyde. Cineole odor was shown to be neutral with regard to the snail's behaviour before learning, because it evokes neither attraction nor avoidance in the free behaving animal (i.e. snails do not move towards or away from it in open-field tests; Nikitin and Balaban, 2000). Therefore, cineole was chosen as a stimulus for associative training.

Three second long odor stimuli were delivered to the nose by a custom-made computer-controlled olfactometer that allowed manipulations of odor concentration in the range of $1-30 \%$ of saturated concentration by changing the ratio of the air flow rate through the odor chamber and that of clean air (Nikitin and Balaban, 2005). Each session started with the delivery of a stimulus with the lowest odor concentration (usually 1\%), followed by stepwise increases in concentration. A second stimulation with the same odor was carried out at least $10 \mathrm{~min}$ after the first one in order to avoid sensory adaptation. The odor chamber contained a filter paper treated with the liquid odorant. Before application to the nose, the two flows were mixed. We measured the airflow with two flowmeters (GF-1100, Gilmont Instruments, Barrington, IL, USA). The total flow was 100-120 ml/ min. When we stimulated the nose with different odor concentrations we maintained the same flow in order to equalize its influence between different trials. Between applications, the odor was continuously removed from the tip of the inner tube of the olfactometer by suction through an outer tube (Figure 1A). Electronically controlled interruption of active odor removal resulted in odor spreading to the nose (details in our previous work: Nikitin and Balaban, 2000; modified from Kauer and Moulton, 1974).

\section{DATA ANALYSIS}

Changes in the frequency and amplitude of oscillatory activity of the PC evoked by odor application were analyzed. The data were normalized; average amplitude or frequency of oscillations in 40-s interval before odor application has been taken as $100 \%$. This allowed us to get a relative mean of the parameter (for instance, relative frequency). Afterwards, the relative means of the parameters in each recording were averaged in 10-s intervals. Such averaging was done to equalize the parameters (amplitude and frequency of oscillations) taken from different experiments, so that the contribution of the different experiments will be equal. The amplitude of each oscillation was measured as a difference between extremes of the ascending and descending phases of the oscillation. The electrophysiological recordings of the oscillatory activity of the $\mathrm{PC}$ were analysed in Spike2 5.0 (CED, UK) and Origin 6.0 (OriginLab, USA).

\section{BEHAVIOURAL EXPERIMENTS (TRAINING SESSIONS)}

To investigate the behavioural significance of odors, we used a conditioning protocol that has been used previously in terrestrial snails for food-aversion conditioning (Balaban et al., 1987), where the snail is tethered by its shell in a manner that allows it to crawl on a ball that rotates freely in water (Figure 1B).

\section{AVERSIVE TRAINING}

Electric shocks $(1-10 \mathrm{~mA}, 0.5 \mathrm{~s}, 10 \mathrm{~Hz})$ were delivered through a macroelectrode applied manually to the left dorsal surface of the 
snail's body. The circuit was completed by the contact of the foot with aluminium foil on the surface of the ball, which in turn was in contact with the grounded salt solution (Figure 1B). The current intensity was adjusted for each snail in such a way that it was just sufficient to cause complete withdrawal of the snail into its shell. Each session started with subthreshold level of stimulation, which was increased stepwise until complete withdrawal was elicited. This amplitude of stimulation was used for the particular snail in this session. No injury to the skin was observed after such electrical stimuli (necrosis is readily seen in snails because of changes in pigmentation and changes in mucus release). The cineole odor source in behavioural tests was placed at a $4-6 \mathrm{~cm}$ distance from the snail, which resulted in a concentration at the location of the animal of $0.1-2 \%$. This concentration was roughly estimated to be inversely proportional to the distance while passively diffusing from the $100 \%$ source. Electric shock (one or two paired trials per day) was delivered $\sim 10 \mathrm{~s}$ after beginning the odor presentation. The experimental group consisted of 20 animals and training lasted for 15-20 days.

\section{FEEDING REINFORCEMENT TRAINING}

To elicit a feeding (approach) reaction of the snail, the same experimental arrangement was used but in this case the cineole odor was paired with carrot juice, which was made fresh every day (Figure 1B). A few drops of carrot juice were delivered to the snail lips $\sim 5 \mathrm{~s}$ after the start of odor presentation. Four or five pairing trials were conducted per day, with at least 15-20 min between pairing trials. The experimental group consisted of 20 animals and training lasted for 15-20 days.

\section{OPEN-FIELD TESTING}

In order to be free of the context reminding about the type of reinforcement (as in electrophysiological experiments), the aversive or feeding reaction to the cineole odor in the snail was tested in an open-field test with double blind control and video taping of the experiment. A glass surface $40 \times 30 \mathrm{~cm}$ divided into squares was used. Each snail was positioned at the beginning of experiment on the midline, and its position was monitored each minute (Figure 1B). The odor source (a vial with filter paper moistened with the odorant, considered as $100 \%$ odor inside the vial) was placed in the middle of the left or right side, so that the shortest distance from the head of the animal to the odor source was $12-14 \mathrm{~cm}$. A gradient of odor concentration was present with maximum near the vial. Each snail was tested in the presence of the odor two to three times, and odor position was alternated for each test. The glass was washed after each snail, and the room was constantly ventilated at a low rate. Maximal testing time in each trial was 6-7 min, less if the snail left the open-field territory. Percentage of time spent at the side with the tested odor served as an estimate of odor aversiveness or attractiveness of the respective odor. It is essential to note that the time spent in the middle $3-\mathrm{cm}$ strip of the open field from which the snail started was not taken into account.

After behavioural testing in an open field, we carried out experiments on semi-intact preparations in which the responses to $1-20 \%$ cineole (delivered using the olfactometer from a distance of $\sim 10 \mathrm{~mm}$ ) were recorded from the PC.

\section{EXPERIMENTS IN VIVO}

In our in vivo experiments, 17 adult snails Helix lucorum and Helix pomatia were used. The snails were kept in an active state, maintained in a humid environment, with the normal day/night light changes (12:12), and were fed cabbage.

Before dissection, the animals were anaesthetized by cooling (during $20 \mathrm{~min}$ ), injected with succinylcholine chloride (Sigma, USA) and solution of $\mathrm{MgCl}_{2}(200 \mathrm{mM}, 2 \mathrm{ml})$. Succinylcholine chloride was dissolved in magnesium chloride in concentration $0.012 \mathrm{mg} / \mathrm{g}$ of the snail's weight (Balaban and Chase, 1989).

The platinum-iridium electrode (A-M Systems, Inc, USA) isolated by teflon $(d \sim 20 \mu \mathrm{m})$ was fixed on the PC covered by the connecting tissue through the slit $(1-1.2 \mathrm{~cm})$ on the skin behind the ommatophores. The teflon isolation was removed from the recording part of the electrode. The position of the recording part of the electrode was close to apical or central part of the PC. Afterwards, the skin was sutured and internal pressure restored with injection of saline.

Recording of the procerebral oscillations started 18-24 h after the surgery, when the animal was active. Each animal was dissected after behavioral experiments to verify the electrode position in the brain. Nine snails survived the operation, behavioral experiments and recording, electrodes were not displaced.

Recording of the procerebral activity during odor application was made using extracellular amplifier (model DL302P, NB-Lab, Russia). We stimulated the olfactory epithelium (see description of the odor meter above) with monomolecular odor cineole (Sigma). The snail was on a ball that rotated freely in a $0.01 \%$ solution of $\mathrm{NaCl}$. Each session started with the delivery of a stimulus with the lowest odor concentration (usually 1\%), followed by stepwise increases of the concentration $(2 \%, 5 \%, 10 \%, 20 \%)$ only to actively moving snails with fully extended ommatophores (posterior tentacles). Minimal time between odor stimuli was $10 \mathrm{~min}$ and the duration of the odor stimulus was $3 \mathrm{~s}$.

To analyze the changes in the amplitude or frequency of the procerebral oscillations in freely behaving animal we normalized the frequency and the amplitude in percents from the averaged values of these parameters for a period $20 \mathrm{~s}$ before odor presentations.

Video taping (video camera JVC F1.2) of the snail's behaviour was made synchronously with PC activity recording. Video data (latency of tentacle withdrawal) were analyzed with Pinnacle Studio 9.0. As a control for electrical artefacts in some experiments the recordings from the mantle were made as well.

\section{STATISTICAL ANALYSIS}

Mann-Whitney rank sum test was used to compare performance of two groups of snails, and Wilcoxon signed-rank test was used for comparison of performance of the same group in different contexts. For comparison of several groups of data ANOVA was used.

\section{RESULTS}

\section{SPONTANEOUS PROCEREBRAL OSCILLATIONS}

We recorded the procerebral oscillations from freely moving animals in order to compare with in vitro recordings and to show that in vitro data reflect what is going on in an intact animal. Spontaneous oscillations recorded in vivo differed from the oscilla- 
tions of $\mathrm{PC}$ recorded in vitro. During extracellular recordings from the PC in semi-intact preparations, the oscillations had a relatively constant frequency and amplitude. Extracellular recordings from the freely moving animals revealed frequent and unpredictable changes in the amplitude and frequency of the procerebral oscillations (Figure 2A).

Frequency of the procerebral oscillations in freely moving animals was in most cases significantly greater compared to the semi-intact preparations. The average frequency of the oscillations recorded in vivo was $1.16 \pm 0.08 \mathrm{~Hz}$ (Figure $2 \mathbf{B}$ ), which is twice the average frequency of semi-intact preparations from both naïve snails and animals trained to respond with a feeding or aversive reaction to cineole.

Frequency of the oscillations recorded from the semi-intact preparations of both naïve and trained snails was rarely higher than $1 \mathrm{~Hz}$ and the average frequency of such oscillations was $0.68 \pm 0.09 \mathrm{~Hz}$ in naïve snails and $0.65 \pm 0.05 \mathrm{~Hz}$ in the "feeding" group of snails
(Figure 2B). The average frequency of the procerebral oscillations recorded in vitro from the snails trained to respond with aversive reaction to cineole was $0.6 \pm 0.04 \mathrm{~Hz}$, and it was never larger than $1 \mathrm{~Hz}$ (Figure 2B).

\section{IN VIVO PROCEREBRAL OSCILLATIONS ELICITED BY ODOR APPLICATION}

It is well known that the electrical activity of the snail's PC changes during odor perception by the animal or during stimulation of the olfactory organs with odors in semi-intact preparations (Delaney et al., 1994; Nikitin and Balaban, 1999, 2000; Toda et al., 2000; Cooke and Gelperin, 2001; Murakami et al., 2004).

Increase of the amplitude of the procerebral oscillations recorded in vivo quite often corresponded to fast changes of the head position or tentacle movements of the videotaped snail. Therefore, in each experiment we compared electrophysiological recordings from the PC with the recordings of potential (second implanted electrode) from the mantle to control for possible movement artefacts.

A
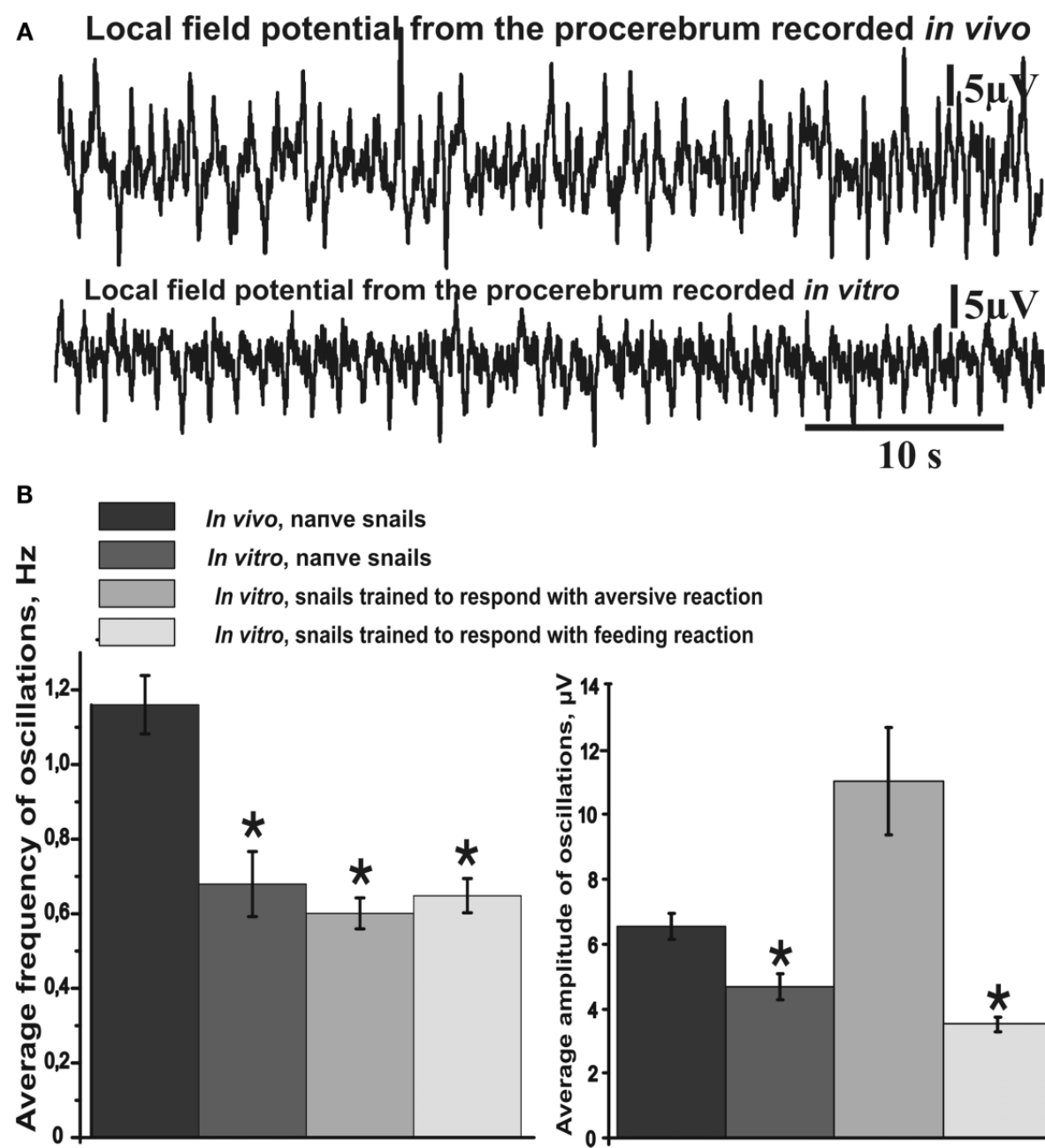

$10 \mathrm{~s}$

FIGURE 2 |Average frequency and amplitude of spontaneous procerebral oscillations during in vivo and in vitro recordings without odor application. (A) Local field potential from the procerebrum. The amplitude and frequency of the procerebral oscillations recorded in vivo undergo frequent spontaneous changes whereas the frequency and amplitude of the oscillations recorded

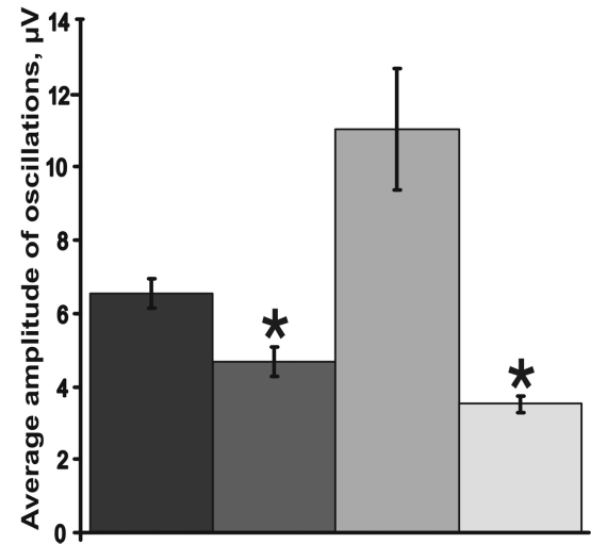

in vitro are more stable. (B) Average frequency and amplitude of the procerebral oscillations recorded in vivo and in vitro without odor stimulation. Average frequency of the procerebral oscillations in vivo without odor stimulation is significantly bigger than the average frequency in semi-intact preparations (asterisks, $p<0.05$, ANOVA, Dunn's method). 
Recordings with significant movement artefacts were not taken for statistical evaluation.

In spite of the observation that the snails in open field do not show escape or approach to cineole odor before training (Nikitin and Balaban, 2000), the short-term tentacle withdrawal reaction to onset of cineol presentation was evident in each experiment, and was described in detail in our previous work (Nikitin and Balaban, 2005).

It is important to note that the tentacle withdrawal in the freely moving snail to cineole application was observed in the first $10 \mathrm{~s}$ only. Maximum of tentacle withdrawal to the $5 \%$ odor application was observed at the 3rd second from the beginning of the odor application. After this a fast tentacle extension took place. The snail's tentacle was almost fully extended by the 9-10th second after the odor application, the sensory epithelium was already exposed by this time, and the animal was able to perceive the odorants again (Figure 3A).

In our experiments it was observed a significant decrease (from $100 \pm 3.68 \%$ to $89.51 \pm 5.8 \%$ ) of the frequency of the procerebral oscillations recorded during the first 10-s time interval after the $5 \%$ cineole application $(p<0.05$, ANOVA with post hoc pairwise multiple comparison, Dunn's method, Figure 3E). A decrease of the oscillation frequency in response to odor corresponded to significant $(p<0.01)$ increase of the amplitude of the procerebral oscillations (from $100 \pm 2.08 \%$ to $112.8 \pm 2.92 \%$, Figure $3 \mathrm{C}$ ).

Changes of the amplitude and frequency of the procerebral oscillations recorded in vivo in the 21-30 and 31-40 s time intervals after $5 \%$ odor application were also significant (Figures 3C,E). Such synchronous changes of the amplitude and frequency of the procerebral oscillations during perception of the odor in low concentration suggest participation of both parameters in the mechanisms of odor perception (recognition, discrimination) by the animal.

Significant changes of the amplitude and frequency of the procerebral oscillations to the $20 \%$ cineole application occurred mostly in the 11-20 s time interval (Figures 3D,F). In this case, the frequency decreased by $12 \%$ in comparison to the baseline (from $100 \pm 3.67 \%$ to $88.59 \pm 6.38 \%, p<0.05$ ), whereas the amplitude $(p<0.01$ ) increased by $25 \%$ (from $100 \pm 2.23 \%$ to $125.22 \pm 6.55 \%)$. It has to be noted that the tentacle withdrawal to the $20 \%$ cineole in the freely behaving animal occurred much faster, and the extension of the tentacle was much slower. Maximal tentacle withdrawal to the $20 \%$ odor application was seen on the 4 th second from the beginning of the odor application, afterwards the slow tentacle extension took place (Figure 3B). It is important to note that the amplitude of maximal tentacle contraction to the $20 \%$ cineole was significantly bigger $(p<0.05$, ANOVA with post hoc pairwise multiple comparison, Dunn's method) at the 4 th-5th second of behavioural response. Obtained results showed that the frequency and amplitude of the procerebral oscillations recorded in vivo undergo synergistic changes: a frequency decrease was accompanied by an amplitude increase in responses to the odor presentation to the snail.

It is important to note that the first reaction of the snail to the odor presentation (fast tentacle withdrawal - quiver, see Nikitin and Balaban, 2005) was similar for both concentrations, while a significant difference in the tentacle contraction dynamics to cineole presentation of different concentrations was observed.

\section{ODOR EVOKED ACTIVITY OF THE PROCEREBRUM IN VITRO}

Changes in amplitude and frequency of the procerebral oscillations after cineole application were calculated in percentages of the mean before (40 s) odor application. Average amplitude of the oscillatory rhythm of the PC in naïve snails in vitro increased during the 21-30 and 31-40 s time intervals after the 5\% cineole application, and was significantly higher in comparison to the baseline. In this case, increase of the amplitude of the procerebral oscillations to a 5\% cineole application was 7\% (from $100.7 \pm 1.44 \%$ to $107.28 \pm 2.26 \%$ ) in the $21-30 \mathrm{~s}$ time interval and $9 \%$ (from $100.7 \pm 1.44 \%$ to $109.47 \pm 2.88 \%$ ) in the $31-40$ s time interval (Figure $4 \mathrm{~A}$ ). No significant changes of the amplitude of the oscillations were observed during the first $20 \mathrm{~s}$ after the odor application to the semi-intact preparation; however the tendency of the amplitude of the oscillations to decrease was obvious (Figure 4A).

A significant decrease in the frequency of the oscillations $(p<0.01)$ was seen during the $1-20$ s time interval to the $5 \%$ cineole application. The decrease in the frequency (from 100.56 $\pm 3.88 \%$ to $79.33 \pm 6.1 \%$ ) was noted right after the odor application (the $1-10 \mathrm{~s}$ time interval). Maximal frequency decrease of $36 \%$ (from $100.56 \pm 3.88 \%$ to $75.56 \pm 6.22 \%$ ) was observed in the $11-20$ s time interval after the $5 \%$ cineole application (Figure 4C); at the same time the tendency of the amplitude to decrease was also obvious.

Cineole in high concentration (20\%) evoked significant $(p<0.001)$ decrease of the amplitude of the procerebral oscillations (from $102 \pm 1.34 \%$ to $90.5 \pm 2.65 \%$ ) as well as the frequency of the oscillations (from $99.8 \pm 4.19 \%$ to $59.24 \pm 6.37 \%$ ) in the $11-20 \mathrm{~s}$ time interval (Figures 4B,D). Frequency of oscillations returned to normal only $30 \mathrm{~s}$ after the odor application to the semi-intact preparations what approximately corresponds to duration of tentacle contraction to same stimulus (Figure 3B).

Working with the semi-intact preparations, we had the chance to record the intracellular activity of the neurons involved in feeding (serotonergic metacerebral neuron $1, \mathrm{MtCl}$, a modulatory neuron of feeding behavior) and aversive (metacerebral neuron 3, MtC3, a motoneuron of tentacle withdrawal) behaviours. Changes in activity of these neurons to the odor application were recorded simultaneously with the extracellular activity of the PC. The MtC3 cells (ommatophore motorneurons involved in tentacle contraction) responded to odor stimuli (cineole) in a concentration-dependent manner: a stronger MtC3 discharge was visible with increased odor concentrations (Figures 4E,F). Meanwhile, the MtC1 (modulatory neuron of the feeding behaviour) responded only to the high concentration odor stimuli. $\mathrm{MtC} 1$ responses were also reflected in the activity of the cerebro-buccal connective because it contains the MtC1's axons (Figure 4F).

\section{Behavioural training}

Procerebrum is responsible not only for perception and recognition, but was shown to be necessary for memory and storage of information about odors (Kasai et al., 2006). It was shown that the changes in procerebral oscillations characteristics to the same odor stimulus can be different in naïve and in trained animals (Kimura et al., 1998; Nikitin and Balaban, 2000).

In our subsequent experiments we trained the snails to respond with the feeding or aversive reaction to the cineole application in order to determine which of the PC reactions corresponds to 


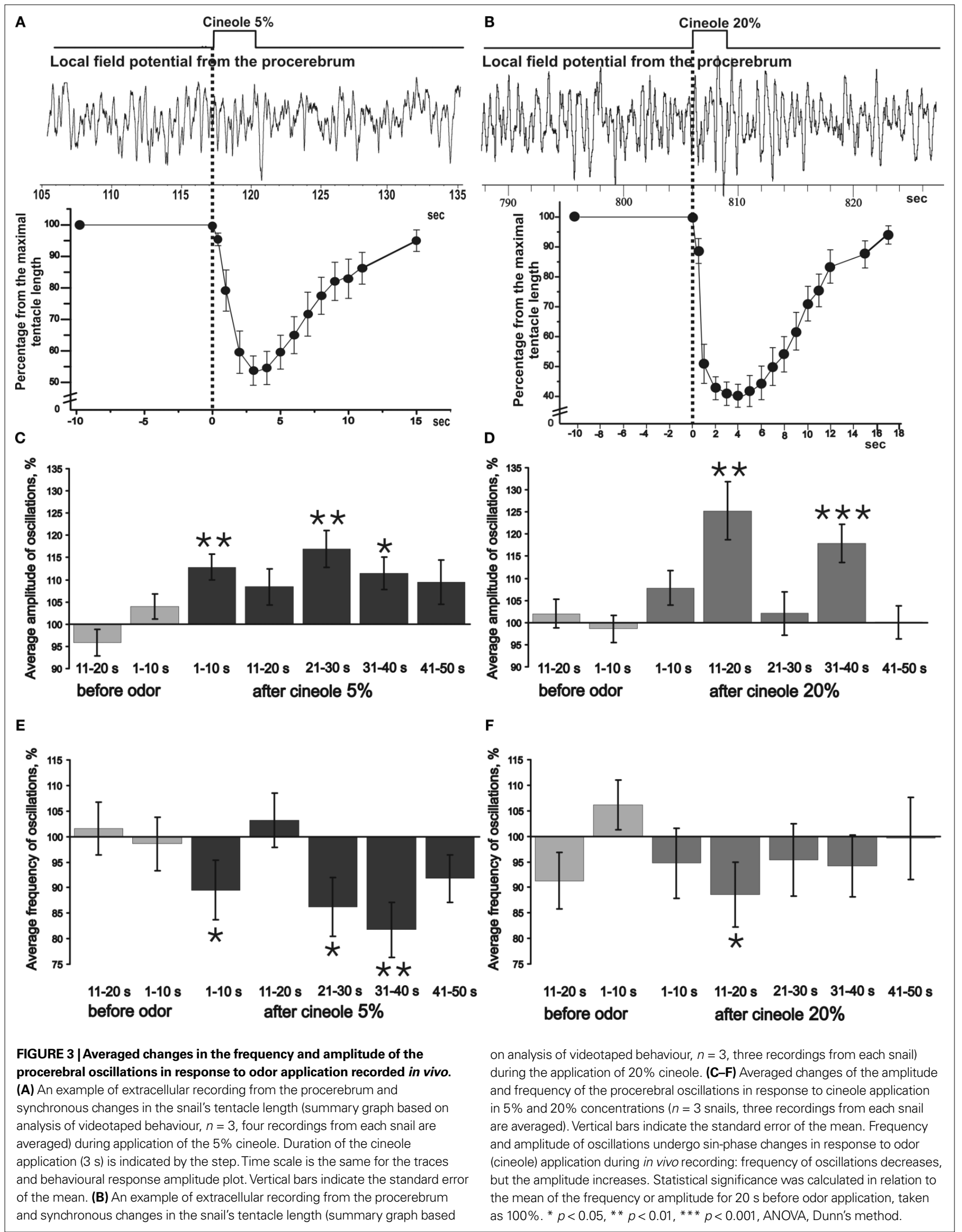




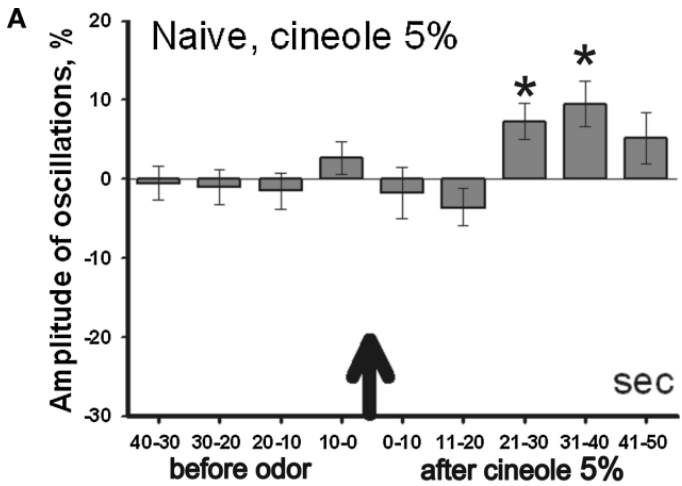

C

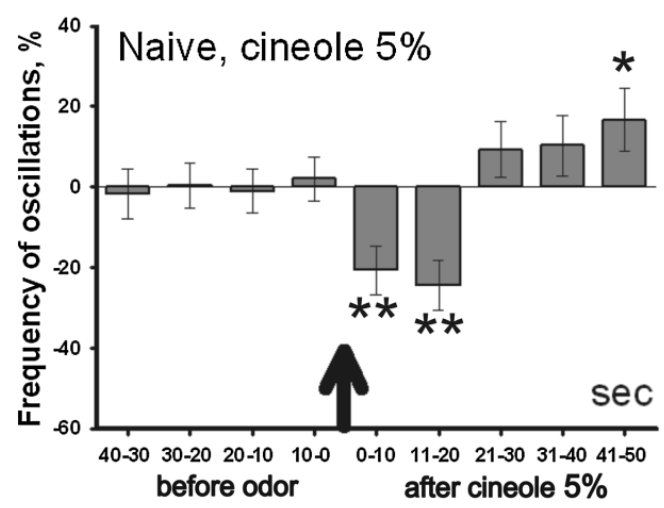

E

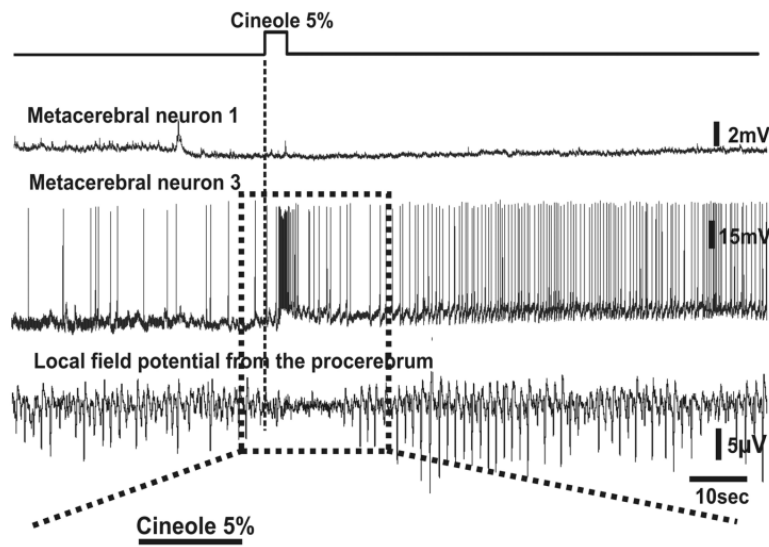

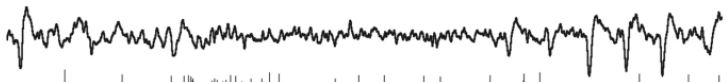

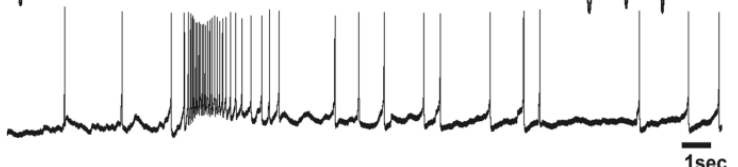

D
B $\left.\circ{ }^{20}\right]$ Naive, cineole $20 \%$
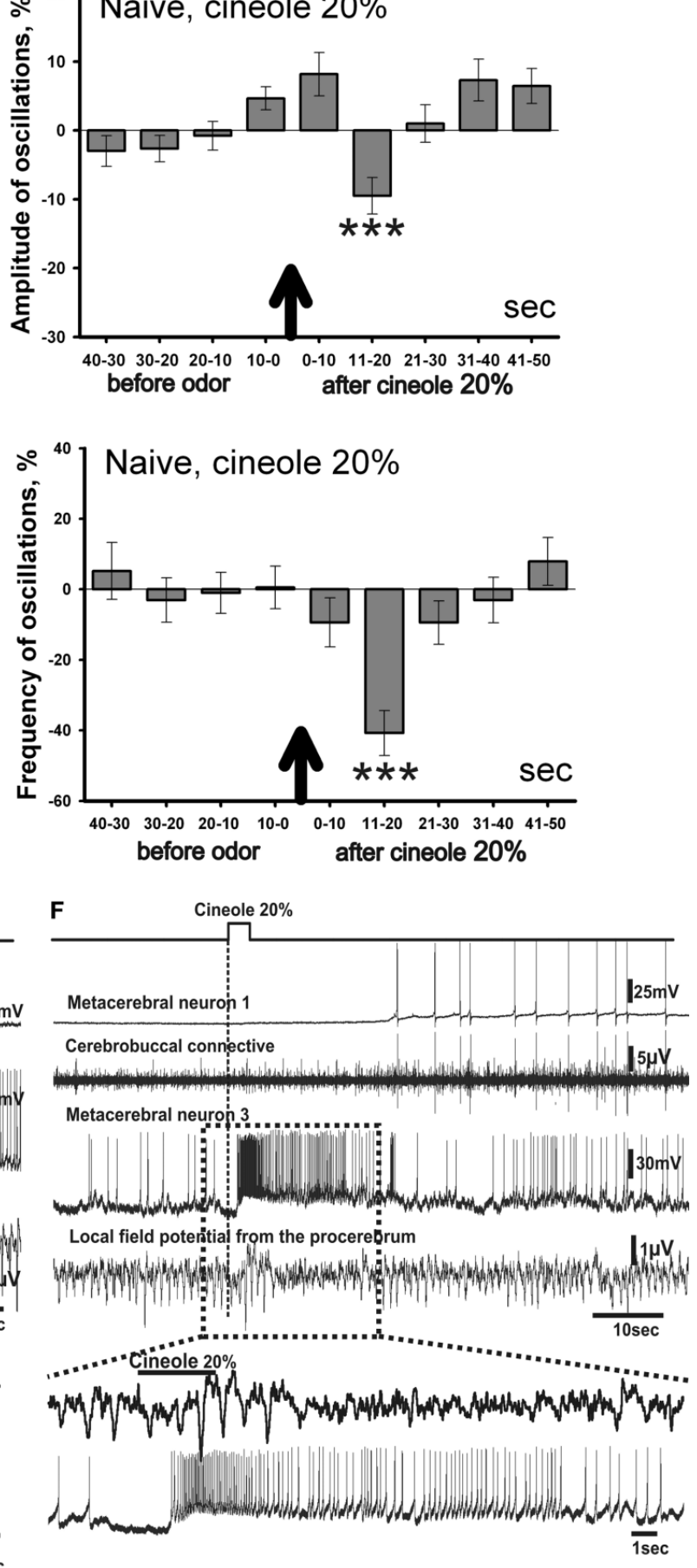

FIGURE 4 | Averaged changes of the oscillatory rhythm recorded in vitro in the procerebrum of naïve snails in response to odor application. (A) Changes in the amplitude of the procerebral oscillations in response to the cineole application in 5\% $(n=5)$ and (B) 20\% ( $n=6)$ concentrations. (C) Changes in the frequency of the procerebral oscillations in response to cineole application in $5 \%(n=5)$ and (D) 20\% (n=6) concentrations. Vertical bars indicate the standard error of the mean. Statistical significance here and on further figures was calculated in relation to the mean of the frequency or amplitude for $20 \mathrm{~s}$ before odor application taken as $100 \% .{ }^{*} p<0.05,{ }^{* *} p<0.01,{ }^{* * *} p<0.001$, ANOVA, Dunn's method. (E,F) The response of the procerebrum, MtC3, MtC1 neurons and the cerebro-buccal connective to $5 \%$ (E) and $20 \%$ (F) cineole application to the nose-brain preparation of naïve snail. Only one example is shown, traces below here and on further figures show part of the response at another time scale. (E) A decrease in the amplitude and frequency of the procerebrum oscillations was observed in semi-intact preparation of the naive snail. No depolarization in $\mathrm{MtC} 1$ neuron after odor presentation was noted. Moderate intensity reaction of $\mathrm{MtC} 3$ neuron to cineole was observed. (F) A decrease in the amplitude and frequency of PC oscillations was observed in semi-intact preparation of the naïve snail in response to $20 \%$ cineole application. Depolarization in $\mathrm{MtC} 1$ neuron after odor presentation is evident. 
positive (approach) or negative (aversion) behavioural response to the same odor. That would allow us to determine the difference between responses to same odor in situation when it is percepted as positive or negative.

Previously it was shown that the cineole odor in the open-field tests do not elicit approach or escape of the snails (Nikitin and Balaban, 2000), and we used this odor in combination with electric shock or food. A gradient of odor concentration was present with maximum near the vial with filter paper moistened with the liquid odorant. Before training, the snails spent $43 \pm 9.6 \%$ of time at the ipsilateral to the presented odor half, and $32 \pm 8.4 \%$ of time in the contralateral half of the open field (non-significant, $p<0.2$, Wilcoxon signed-rank test). The snails trained to respond aversively to the cineole application (odor + electric shock) demonstrated long-lasting tentacle withdrawal, slowing down of the locomotion and turning the head away from the odor source. These snails tried to avoid the odor source in open-field tests (Figure 1B) by moving to the other side of the field or hiding inside the shell. Percentage of time spent in the opposite to the odor half of the field in aversively trained snails was $48.3 \pm 10.6 \%$, while at the half with odor the same snails spent only $4.6 \pm 3.1 \%$ of time ( $n=18$ snails, $p<0.001$, Wilcoxon signed-rank test). The rest of time the snails spent at the middle strip of the open field.

The snails trained to respond with the feeding reaction (odor + food) to the cineole revealed their preference for this odor in open-field tests by moving in direction of the source of the conditioned odor. They spent $56.8 \pm 3.9 \%$ of time at the half with odor, while the same snails spent only $5.6 \pm 3.4 \%$ at the opposite to odor half of the field ( $n=16, p<0.001$, Wilcoxon signed-rank test). The rest of time the snails spent at the middle strip of the open field.

Obtained results clearly showed that associative training resulted in statistically significant preference of the odor after the training with food reinforcement (positive response), and aversive behavioural response after training with noxious reinforcement.

\section{PROCEREBRAL RESPONSES TO THE CINEOLE APPLICATION IN AVERSIVELY CONDITIONED SNAILS}

Dynamics of changes of the oscillatory rhythm recorded in vitro in the PC of snails trained to respond with the aversive reaction to the cineole had a characteristic pattern.

A significant $(p<0.001)$ short-term decrease of the amplitude of the procerebral oscillations $(16 \%)$ was observed in response to cineole in low concentration (5\%) in the 11-20 s time interval after odor application (Figure 5A). In the 21-30 s time interval a decrease of the amplitude of the procerebral oscillations was also observed. Afterwards, the amplitude of the procerebral oscillations returned to the baseline (Figure 5A).

Changes of the frequency of the procerebral oscillations in response to the cineole application in low concentrations were more prolonged but occurred at the same time intervals as the changes of the amplitude of oscillations. A significant $(p<0.01)$ decrease of the frequency of the procerebral oscillations can be noticed in the first 10-s time interval after odor presentation to the semi-intact preparation. Maximal decrease of the frequency of the oscillations (by 53\%) was observed in the 11-20 s time interval after $5 \%$ odor presentation (Figure 5C), i.e. at the same period when the maximal decrease of the amplitude of oscillations (16\%) took place (Figure 5A). Decrease of frequency as well as of amplitude of the procerebral oscillations was observed in the 21-30 s time interval. Afterwards, frequency and amplitude of the procerebral oscillations returned to baseline.

Decrease (from $98.28 \pm 1.75 \%$ to $75.29 \pm 2.17 \%$ ) in the frequency of the procerebral oscillations was evoked by $20 \%$ cineole in the 11-20 s time interval after odor presentation (Figure 5B). This reaction of the $\mathrm{PC}$ was synchronized with the decrease in the amplitude of the oscillations during the same time interval, but in this case the amplitude of the oscillations decreased by half (from $100.31 \pm 5.62 \%$ to $45.36 \pm 6.72 \%$, Figure 5D).

A significant decrease in the frequency (26\%) and amplitude $(9 \%)$ of the procerebral oscillations to the $20 \%$ cineole was observed during the 21-30 s time interval after cineole application (Figures 5B,D). Afterwards, both frequency and amplitude quickly returned to the baseline levels.

Changes in the neuronal activity in response to the cineole application appeared mostly in the ommatophore motoneuron (MtC3). Reactivity of this neuron to the cineole application was similar regardless of the odor concentration (Figures 5E,F). Essential changes of the activity in other recorded neurons were not registered. Such a long and strong reaction of the MtC3 neuron to the cineole is obviously linked with the aversiveness of this odor for the animal. Desynchronization of the procerebral oscillations in response to the odor application is obvious after the cineole application.

It is important to note that the PC of naïve snails and snails trained to respond with an aversive reaction to the cineole both reacted to the high concentration cineole $(20 \%)$ with a decrease in the frequency of the oscillations (Figures 4D and 5D). It is interesting that the group of snails trained to respond with aversive reaction had a more apparent decrease in the frequency of the oscillations (Figure 5D). This could be an evidence of the aversiveness of the highly concentrated odor for both the naive and trained snails.

\section{PROCEREBRAL RESPONSES TO THE CINEOLE APPLICATION IN POSITIVELY CONDITIONED SNAILS}

The procerebral oscillations in preparations from snails trained to respond with positive (approach) reaction to the cineole odor demonstrated a significant decrease in the amplitude of the oscillations to the odor of low concentration (5\%) during the first $10 \mathrm{~s}$ after cineole presentation (Figure 6A). The amplitude of the procerebral oscillations returned to normal $10 \mathrm{~s}$ after odor presentation to the semi-intact preparation.

Along with the amplitude, the frequency of the oscillations had changed with the cineole application of $5 \%$ concentration. A significant decrease in the frequency $(41 \%)$ of the procerebral oscillations occurred during the first $10 \mathrm{~s}$ after odor presentation to the semi-intact preparation (Figure 6C), after that the frequency of the oscillations quickly returned to baseline. Hence, changes in the amplitude and frequency of the procerebral oscillations in the semi-intact preparations of the snails trained to respond with the positive reaction to the cineole occurred simultaneously.

Such an early reaction (during the first 10-s time interval) of the PC to the cineole (5\%) application is an important difference 


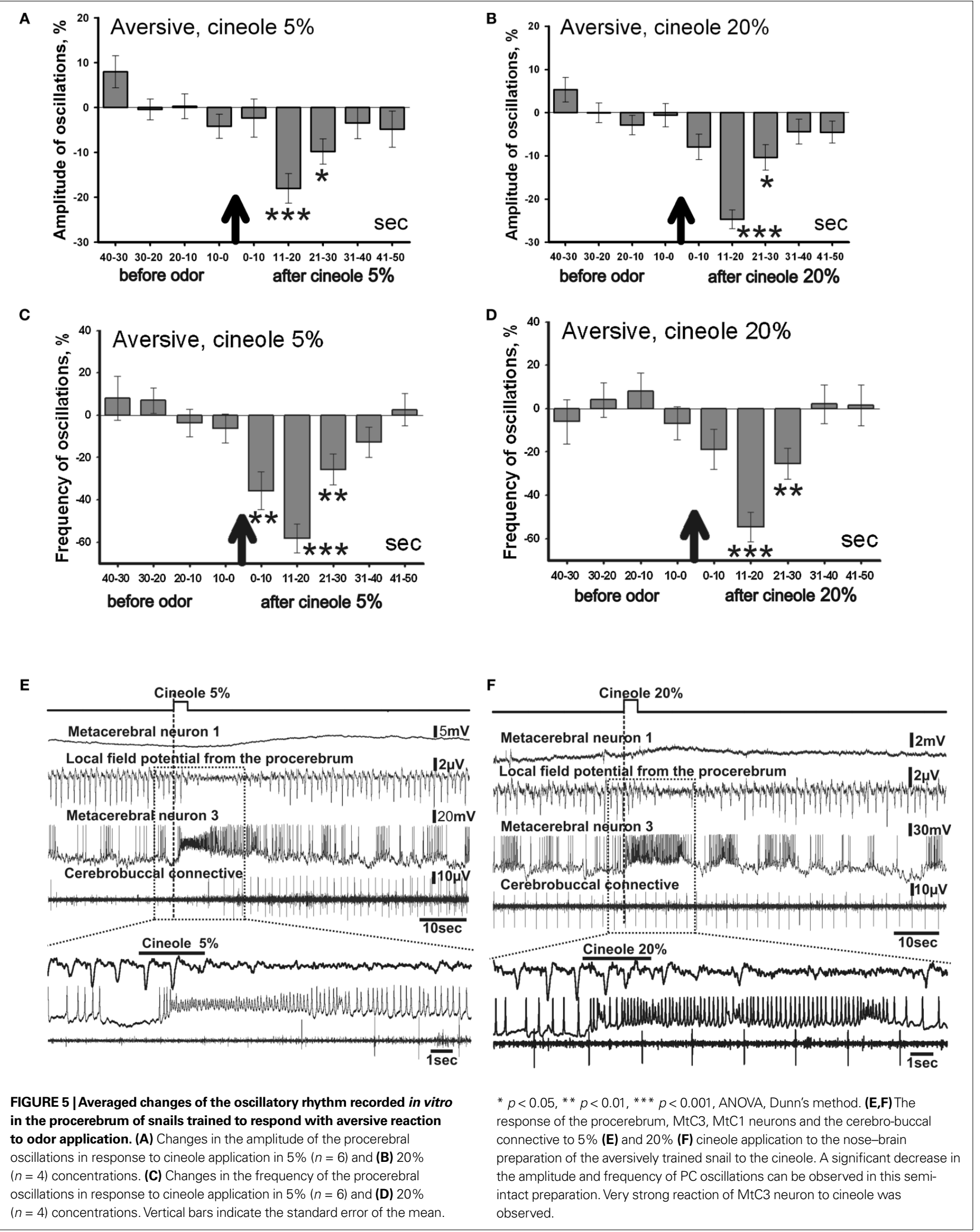




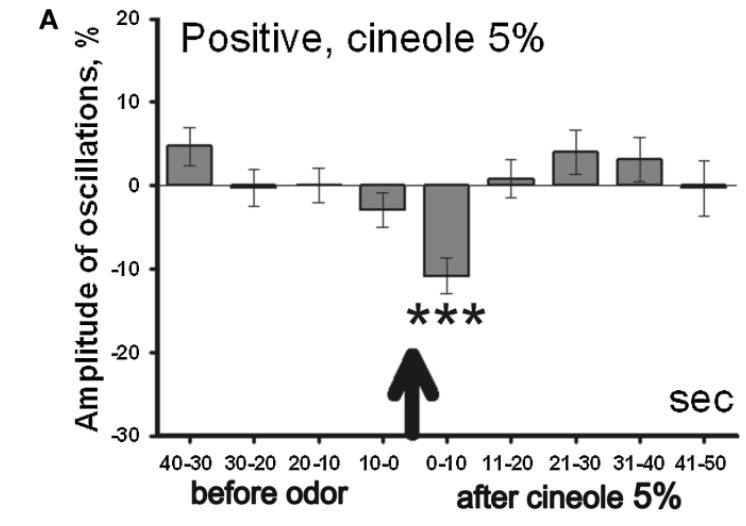

C

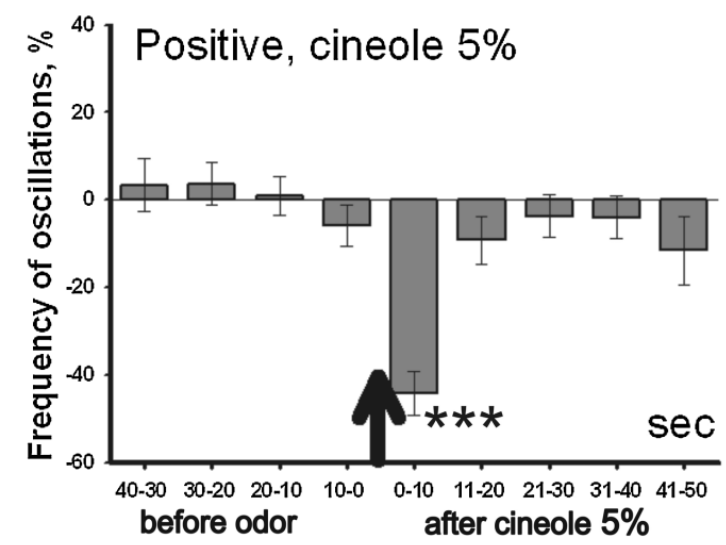

E

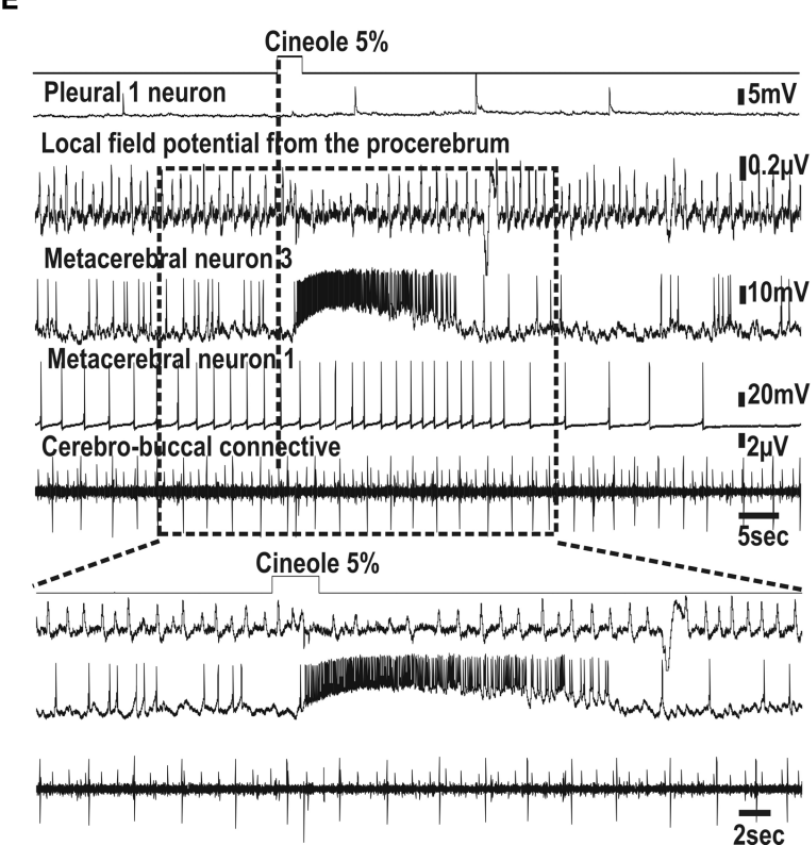

FIGURE 6 | Averaged changes of the oscillatory rhythm recorded in vitro in the procerebrum of snails trained to respond with feeding reaction to odor application. (A) Changes in amplitude of the procerebral oscillations in response to cineole application in $5 \%(n=6)$ and (B) $20 \%$ $(n=5)$ concentrations. (C) Changes in frequency of the procerebral oscillations in response to cineole application in $5 \%(n=6)$ and (D) $20 \%$
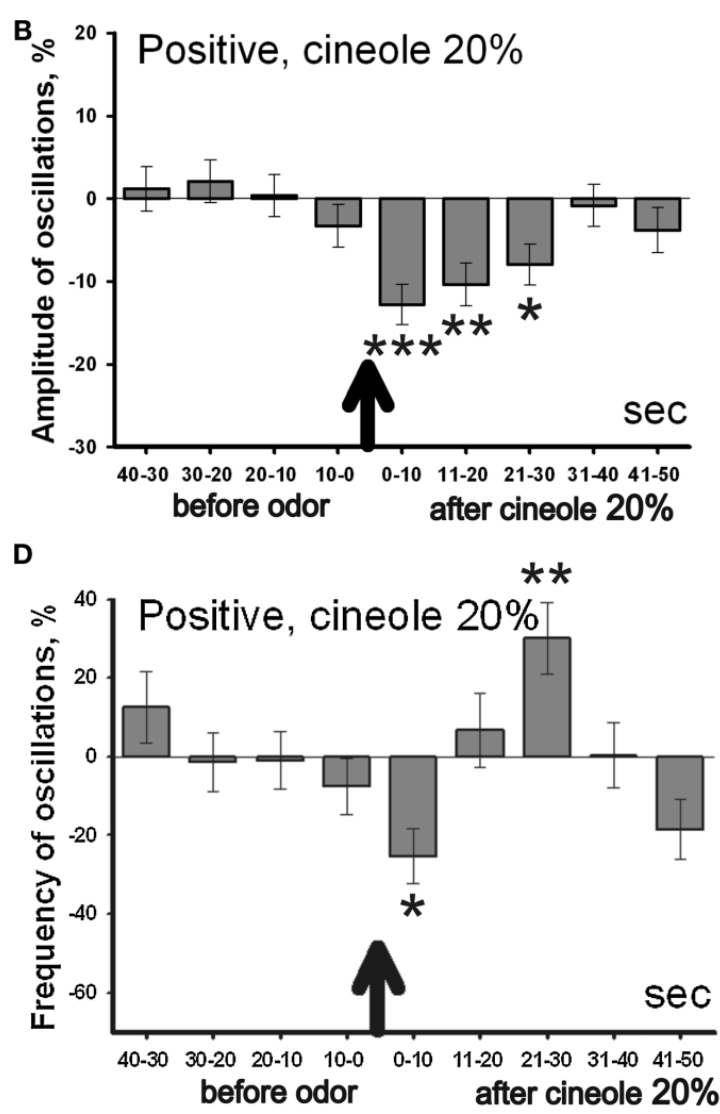

$\mathbf{F}$

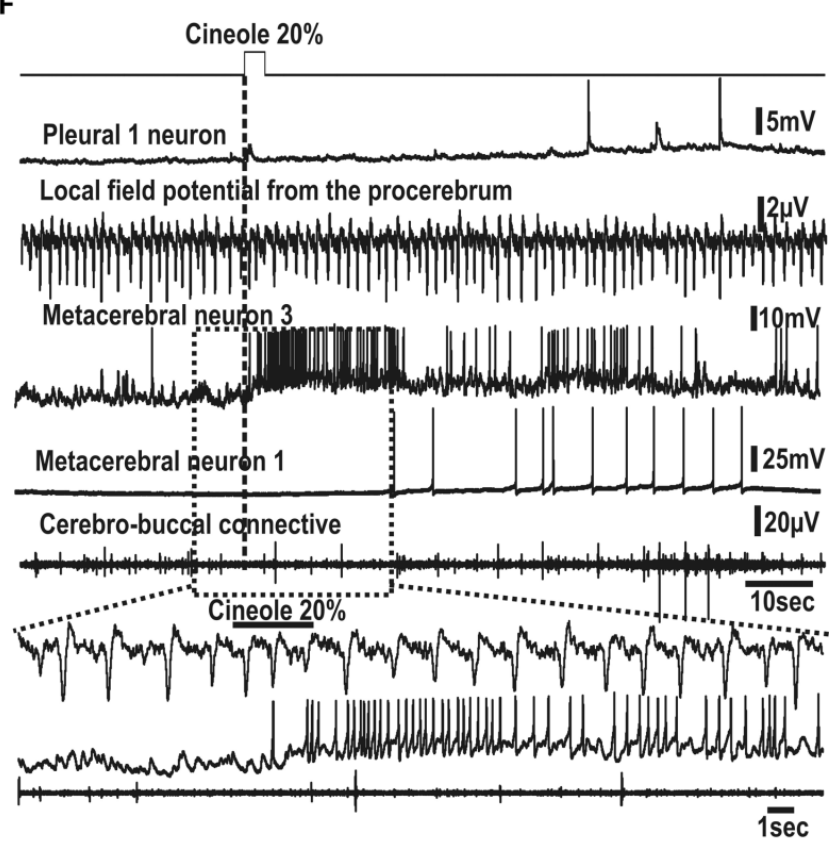

$(n=5)$ concentrations. Vertical bars indicate the standard error of the mean. ${ }^{*} p<0.05,{ }^{* *} p<0.01,{ }^{* *} p<0.001$. (E, F) The response of the procerebrum, $\mathrm{MtC} 3, \mathrm{MtC} 1$ neurons and the cerebro-buccal

connective to cineole $5 \%$ (E) and $20 \%$ (F) application to the nose-brain preparation of the snail trained to respond with the feeding reaction to the cineole. 
from the procerebral reactions of the naïve snails and snails trained to respond with negative behavioural reaction to the cineole.

In response to the high concentration of cineole (20\%), changes in the amplitude of the procerebral oscillations were more prolonged in this group of snails. Significant decrease of the amplitude of the procerebral oscillations was recorded during the first $30 \mathrm{~s}$ after the odor presentation to the semi-intact preparation (Figure 6B). However, the maximum decrease $(11 \%)$ in the amplitude of the oscillations was also observed during the first $10 \mathrm{~s}$ after odor application. The amplitude of the oscillations returned to the baseline levels $30 \mathrm{~s}$ after odor application.

Frequency of the procerebral oscillations decreased nonsignificantly during the first $20 \mathrm{~s}$ after the $20 \%$ odor application in the snails trained to respond with the positive reaction. However, a significant increase (30\%) of the oscillations frequency occurred $20 \mathrm{~s}$ after the odor presentation (Figure 6D). Frequency increase was never observed in aversively trained snails.

Along with the changes of the procerebral activity, the reaction of identified central neurons to the cineole application was recorded in the snails of this group. Reaction of the ommatophore motoneuron ( $\mathrm{MtC} 3$ ) was not as prolonged as in aversively trained snails to both the $5 \%$ and $20 \%$ cineole concentrations, indicating a shorter tentacle withdrawal. Such changes in neural activity can be a result of association of feeding reinforcement with cineole application in these snails (Figures 6E,F). At the same time, the $\mathrm{MtC} 1$ neuron and the cerebro-buccal connective recordings showed spike activity in response to the high concentration (20\%) cineole application, which may be due to the activation of the feeding behaviour by the snail.

Changes in the frequency and amplitude of the oscillations in the snails trained to respond with the positive reaction to the $5 \%$ cineole application appeared to be relatively short-term, while the $20 \%$ cineole evoked more prolonged and changing the sign (from decrease to increase) responses in the PC.

\section{COMPARATIVE ANALYSIS OF RESPONSES TO CINEOLE IN NAÏVE AND TRAINED SNAILS}

In order to understand better the observed changes we plotted all data with the same scale (Figure 7). It is a common assumption that responses depend on the concentration of the cineole. In conformity with this assumption, the amplitude and frequency of the procerebral oscillations in naïve snails only slightly changed in response to the $5 \%$ cineole (Figures 7A,C), whereas in response to $20 \%$ cineole the changes were more expressed and significant (Figures 7B,D).

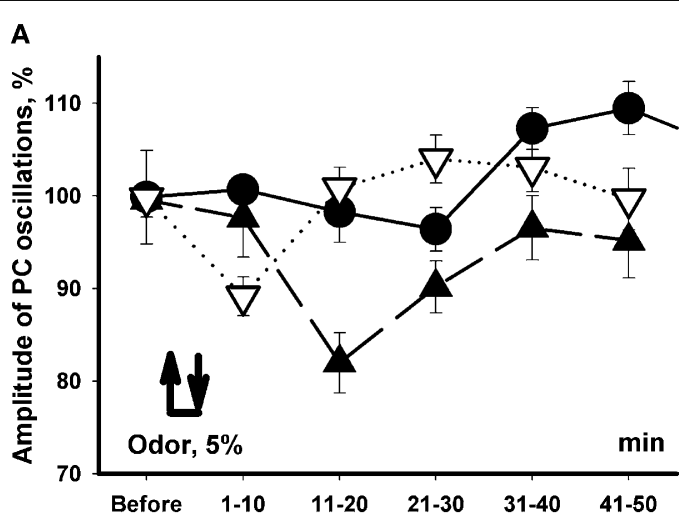

C

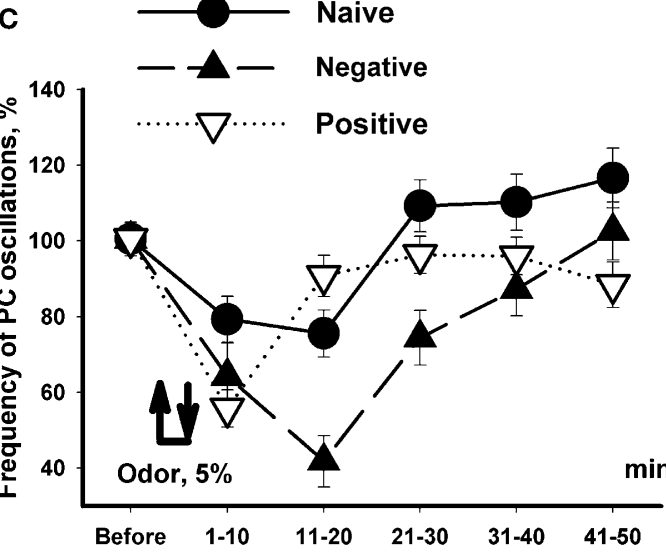

FIGURE 7 | Changes in the amplitude and frequency of oscillations in the procerebrum recorded in vitro in response to $5 \%(\mathrm{~A}, \mathrm{C})$ and $20 \%(\mathrm{~B}, \mathrm{D})$ cineole application. Vertical bars indicate the standard error of the mean

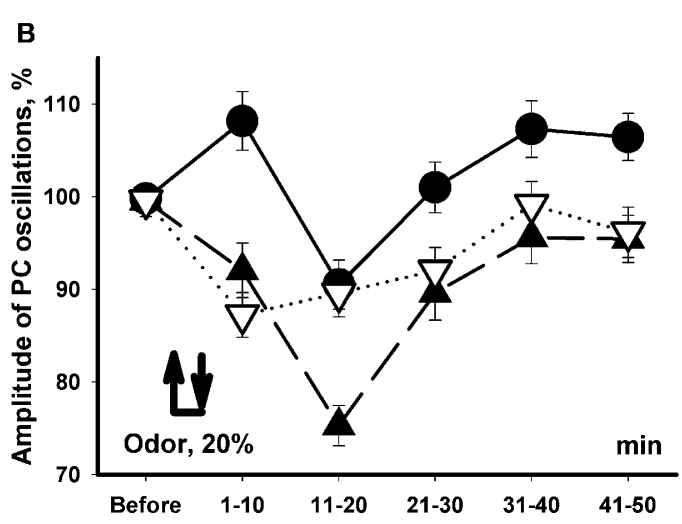

D

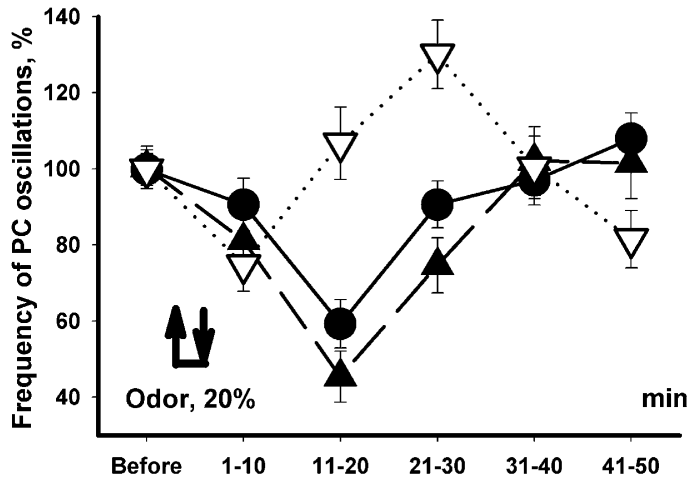

(asterisks, $p<0.05$, ANOVA, Dunn's method). The reactions of the snails trained to respond with aversive or feeding reaction to cineole were significantly different. 
Unexpectedly, the snails trained to respond with negative (escape) reaction to the cineole showed almost the same dynamics of changes in amplitude and frequency to the cineole application regardless of the concentration of the odor (Figure 7). It is important to note that the responses of the snails trained to be aversive differed in amplitude and frequency compared to naïve snails only in the 11-20 and 21-30 s time intervals in response to $5 \%$ cineole application on the semi-intact preparations $(p<0.02$, ANOVA, Dunn's method, Figures 7A,C). A significant $(p<0.05$, ANOVA, Dunn's method)) difference in amplitude of the procerebral oscillations was found between naïve and aversively trained snails in each 10-s time interval when exposed to the cineole in $20 \%$ concentration (Figure 7B). On the other hand, the dynamics of changes in frequency of oscillations was very similar in naïve and aversively trained snails in response to the high concentration of odor (Figure 7D), what reflects a relatively bigger changes in naive snails to the $20 \%$ cineole.

In the first 10-s time interval after odor application, the changes in frequency of the oscillations similar in direction to changes in naïve snails were observed in positively trained snails. After this a fast increase in the frequency of the oscillations similar to changes in naïve snails took place (Figure 7C). Interestingly, in response to $20 \%$ cineole, the frequency of procerebral oscillations significantly ( $p<0.001$, ANOVA, Dunn's method) increased in the positively trained group of snails compared to the naïve snails in the 11-20 and even more in the 21-30 s time intervals after odor application (Figure 7D). No significant changes in the amplitude of procerebral oscillations of the positively trained snails were found (Figure 7A).

Obtained results suggest existence of significant changes in responsiveness in snails trained to approach or escape the same odor. Maximal difference ( $p<0.001$, ANOVA, Dunn's method) was observed in the interval 10-30 s after the beginning of odor application (Figure 7D).

\section{DISCUSSION}

Relation of the PC oscillations to odor recognition/categorization in terrestrial snails is controversial. In vitro it was shown that not only noxious, but food odors also can elicit a temporary decrease with following increase of frequency (Nikitin and Balaban, 2000). The $\mathrm{PC}$ responses are not stable and vary significantly during the experiment (Nikitin and Balaban, 1999). Those were the reasons for us to undertake recordings from freely behaving snails. Our results clearly show that during a stage of tentacle withdrawal to odor presentation, a significant decrease of PC oscillations frequency is taking place (Figure 3). Concomitant increase of amplitude may reflect synchronization of procerebral neural activity.

Comparison of the $\mathrm{PC}$ responses to odors in vivo and in vitro revealed certain similarities and differences. In in vivo recordings a decrease in frequency of PC responses to cineole was accompanied by an increase in amplitude (Figure 3), while in in vitro preparations (Figures 4-7) a decrease in frequency was accompanied by a decrease in amplitude. We think that the frequency of oscillations is a more informative component of odor information processing than the amplitude, which depends on the recording techniques. In our experiments, the difference in recording techniques (platinumiridium wire in in vivo and glass electrode filled with saline in in vitro experiments), and consequent difference in areas of recording do not allow us to make any conclusions about the difference in changes of the amplitude of PC oscillations.

Responses of the PC of the aversively trained snails to the cineole were generally similar in direction to the responses of the naïve snails, but changes of the amplitude and frequency of the oscillations in the "aversive" group of snails were much more pronounced. No significant difference in responses to $5 \%$ and $20 \%$ cineole was noted. In a recent paper in terrestrial slug Limax (Inoue et al., 2006), the PC responses to aversively conditioned odor consisted of long-term (tens of seconds) $20-30 \%$ increase in oscillation frequency in in vitro preparation, similar to the responses to noxious odors. Unfortunately, the duration of odor presentation in these experiments was significantly higher $(3 \mathrm{~s}$ in our experiments, and 15 or $20 \mathrm{~s}$ in experiments of Inoue et al.), making it impossible to compare these results with the responses to aversive stimuli in our experiments in which a transient decrease in frequency was observed.

In behavioral experiments it was clearly observed (Nikitin and Balaban, 2005; Nikitin et al., 2008), that all snails (including ones trained to respond with a feeding reaction) withdrew their tentacles during the first few seconds after the odor in any concentration was presented. After the withdrawal, the snails trained to respond positively protracted the tentacles and moved actively towards the odor source, while the snails trained to respond with aversion continued to contract the tentacles, withdrew the head. It is clearly seen in Figure 7 that the decrease in frequency was very short in the snails trained to respond positively relatively the responses of the snails trained aversively, and even a significant increase of frequency was observed in the interval 20-30 s after the odor presentation in positively trained snails.

Changes in the spontaneous oscillations frequency in the snails trained to respond with the positive reaction to cineole depended on the concentration of the applied odor, and these responses were qualitatively similar to responses of other groups during first $10 \mathrm{~s}$ of response to odor (tentacle quiver in all groups of animals), but significantly different from the responses of the aversively trained and naïve snails in the interval 11-30 s (Figures 4-7). The main difference (increase in PC oscillations frequency) was observed in the 11-30 s time interval after the odor presentation, what corresponds to the end of the tentacle quiver and timing of decision making (approach or escape) in the free behaving snails.

Assuming that the changes in the oscillatory activity of the PC in snails trained to respond with feeding or aversive reactions are linked with the realization of different types of behaviour, it is logical to assume that the decrease of frequency of the procerebral oscillations observed during first 10 s indicates an aversive reaction, while increase in frequency correlate with extension of tentacles and approach to food.

In literature many authors discuss an opinion that frequency and amplitude of the PC oscillations encode different information about the odor (Cooke and Gelperin, 2001; Ermentrout et al., 2001). This hypothesis does not contradict to our results. The desynchronization (the amplitude and frequency decrease) of the activity of the procerebral neurons observed during first $10 \mathrm{~s}$ of response thus should not be connected to the odor perception, while the synchronization and the increase of the frequency observed during 10-30th seconds 
correlate to establishment of the behavioral validity of odors and start of the food search (feeding behaviour).

Obtained results suggest involvement of the procerebral neurons in behavioral decision to approach or escape the source of odor.

\section{REFERENCES}

Balaban, P. M. (1993). Behavioral neurobiology of learning in terrestrial snails. Prog. Neurobiol. 41, 1-19.

Balaban, P. M. (2002). Cellular mechanisms of behavioral plasticity in terrestrial snail. Neurosci. Biobehav. Rev. 26, 597-630.

Balaban, P. M., and Chase, R. (1989). Self-stimulation in snails. Neurosci. Res. Commun. 4, 139-146.

Balaban, P. M., Vehovszky, A., Maximova, O. A., and Zakharov, I. S. (1987). Effect of 5,7-dihydroxytryptamine on the food-aversive conditioning in the snail Helix lucorum L. Brain Res. 24, 404, 201-210.

Buck, L., and Axel, R. (1991). A novel multigene family may encode odorant receptors: a molecular basis for odour recognition. Cell 65, 175-187.

Chase, R. (1985). Responses to odours mapped in snail tentacle and brain by $\left[{ }^{14} \mathrm{C}\right]$-2-deoxyglucose autoradiography. J. Neurosci. 5, 2930-2939.

Chase, R., and Croll, R.P.(1981). Tentacular function in snail olfactory orientation. J. Comp. Physiol. 143, 357-362.

Chase, R., and Tolloczko, B. (1986). Synaptic glomeruli in the olfactory system of a snail, Achatina fulica. Cell Tissue Res. 246, 567-573.

Chase, R., and Tolloczko, B. (1993). Tracing neural pathways in snail olfaction: from the tip of the tentacles to the brain and beyond. Microsc. Res. Tech. 24, 214-230.

Cooke, I. R., and Gelperin, A. (2001). In vivo recording of spontaneous and odor-modulated dynamics in the Limax olfactory lobe. J. Neurobiol. 46, 126-141.

Delaney, K. R., Gelperin, A., Fee, M. S., Flores, J. A., Gervais, R., Tank, D. W., and Kleinfeld, D. (1994). Waves and stimulus-modulated dynamics in an oscillating olfactory network. Proc. Natl. Acad. Sci. U.S.A. 91, 669-673.

Dorries, K. M., and Kauer, J. S. (2000). Relationships between odor-elicited oscillations in the salamander olfactory epithelium and olfactory bulb. J. Neurophysiol. 83, 754-765.
Egan, M. E., and Gelperin, A. (1981). Olfactory inputs to a bursting serotonergic interneuron in a terrestrial mollusc. J. Molluscan Stud. 47, 80-88.

Ermentrout, B., Wang, J. W., Flores, J., and Gelperin,A. (2001). Model of olfactory discrimination and learning in Limax procerebrum incorporating oscillatory dynamics and wave propagation. J. Neurophysiol. 85, 1444-1452.

Gelperin, A., and Tank, D. W. (1990). Odour-modulated collective network oscillations of olfactory interneurons in a terrestrial mollusc. Nature 345, 437-440.

Gervais, R., Kleinfeld, D., Delaney, K. R., and Gelperin, A. (1996). Central and reflex neuronal responses elicited by odour in a terrestrial mollusc. J. Neurophysiol. 76, 1327-1339.

Gray, C. M., and Skinner, J. E. (1988). Centrifugal regulation of neuronal activity in the olfactory bulb of the walking rabbit as revealed by reversible cryogenic blockade. Exp. Brain Res. 69, 378-386.

Ierusalimsky, V. N., and Balaban, P. M. (2007). Primary sensory neurons containing command neuron peptide constitute a morphologically distinct class of sensory neurons in the terrestrial snail. Cell Tissue Res. 330, 169-177.

Inoue, T., Murakami, M., Watanabe, S., Inokuma, Y., and Kirino, Y. (2006). In vitro odor-aversion conditioning in a terrestrial mollusk. J. Neurophysiol. 95, 3898-3903.

Kasai, Y., Watanabe, S., Kirino, Y., and Matsuo, R. (2006). The procerebrum is necessary for odor-aversion learning in the terrestrial slug Limax valentianus. Learn. Mem. 13, 482-488.

Kauer, J. S., and Moulton, D. G. (1974). Responses of olfactory bulb neurones to odour stimulation of small nasal areas in the salamander. J. Physiol. 243, 717-737.

Kimura, T., Toda, S., Sekiguchi, T., and Kirino, Y. (1998). Behavioural modulation induced by food odor aversive conditioning and its influence on the olfactory responses of an oscillatory

\section{ACKNOWLEDGEMENTS}

The work was supported by grants from Russian Foundation for Basic Research, Biology Division of Russian Academy of Sciences, Council for Grants of the President of RF.

brain network in the slug Limax marginatus. Learn. Mem. 4, 365-375.

Kleinfeld, D., Delaney, K. R., Fee, M. S. Flores, J. A., Tank, D. W., and Gelperin A. (1994). Dynamics of propagating waves in the olfactory network of a terrestrial mollusc: an electrical and optical study. $J$. Neurophysiol. 72, 1402-1419.

Lam, Y. W., Cohen, L. B., Wachowiak, M., and Zochowski, M. R. (2000). Odors elicit three different oscillations in the turtle olfactory bulb. J. Neurosci. 20 , 749-762.

Laurent, G., and Davidowitz, H. (1994). Encoding of olfactory information with oscillating neural assemblies. Science 265, 1872-1875.

Mellon, D., and Wheller, C. J. (1999). Coherent oscillations in membrane potential synchronize impulse bursts in central olfactory neurons of the crayfish. J. Neurophysiol. 81, 1231-1241.

Mombaerts, P., Wang, F., Dulac, C. Chao, S. K., Nemes, A. Mendelsohn, M., Edmonson, J., and Axel, R. (1996). Visualizing an olfactory sensory map. Cell 87, 675-686.

Murakami, M., Watanabe, S., Inoue, T. and Kirino, Y. (2004). Odor-evoked responses in the olfactory center neurons in the terrestrial slug. J. Neurobiol. $58,369-378$.

Nikitin, E. S., and Balaban, P. M. (1999). Optical recording of the odorevoked responses in olfactory structures of the brain of the terrestrial mollusk Helix. Zhurn. vyssh.nervn. deyat. (in Russian) 49, 817-829.

Nikitin, E. S., and Balaban, P. M. (2000) Optical recording of odor-evoked responses in the olfactory brain of the naive and aversively trained terrestrial snails. Learn. Mem. 7, 422-432.

Nikitin, E. S., and Balaban, P. M. (2005). Fine tuning of olfactory orientation behaviour by the interaction of oscillatory and single neuronal activity. Eur. J. Neurosci. 22, 2833-2844.

Nikitin, E. S., Korshunova, T. A., Zakharov, I. S., and Balaban, P. M. (2008). Olfactory experience modi- fies the effect of odour on feeding behaviour in a goal-related manner. J. Comp. Physiol. A Neuroethol. Sens. Neural. Behav. Physiol. 194, 19-26.

Ratte, S., and Chase, R. (1997). Morphology of interneurons in the procerebrum of the snail Helix aspersa. J. Comp. Neurol. 384, 359-372.

Sahley, C., Gelperin, A., and Rudy, J. W. (1981). One-trial associative learning modifies food preferences of a terrestrial mollusk. Proc. Natl. Acad. Sci. U.S.A. 79, 640-642.

Toda, S., Kawahara, S., and Kirino, Y (2000). Image analysis of olfactory responses in the procerebrum of the terrestrial slug Limax marginatus. J. Exp. Biol. 203, 2895-2905.

Watanabe, S., Kirino, Y., and Gelperin, A. (2008). Neural and molecular mechanisms of microcognition in Limax. Learn. Mem. 15, 633-642.

Wehr, M., and Laurent, G. (1999). Relationship between afferent and central temporal patterns in the locust olfactory system. J. Neurosci. 19, 381-390.

Conflict of Interest Statement: The authors declare that the research was conducted in the absence of any commercial or financial relationships that could be construed as a potential conflict of interest.

Received: 01 February 2009; paper pending published: 11 June 2009; accepted: 27 July 2009; published online: 27 August 2009.

Citation: Samarova $E$ and Balaban $P$ (2009) Changes in frequency of spontaneous oscillations in procerebrum correlate to behavioural choice in terrestrial snails. Front. Cell. Neurosci. 3:8. doi: 10.3389/neuro.03.008.2009

Copyright (c) 2009 Samarova and Balaban. This is an open-access article subject to an exclusive license agreement between the authors and the Frontiers Research Foundation, which permits unrestricted use, distribution, and reproduction in any medium, provided the original authors and source are credited. 